\title{
Proximal corporal reconstruction: adjunct of penile prosthesis revision surgery
}

\author{
Emad Rajih ${ }^{1,2} \cdot$ Arthur L. Burnett ${ }^{2}$
}

Received: 13 October 2018 / Revised: 29 December 2018 / Accepted: 8 January 2019 / Published online: 31 January 2019

(c) Springer Nature Limited 2019

\begin{abstract}
Proximal corporal deformities may account for failed inflatable penile prosthesis (IPP) surgery. No contemporary series has focused solely on describing the natural history and management of isolated proximal corporal deformities in patients after IPP surgery. The aim of the current report is to present the clinical courses and surgical management with outcomes of proximal corporal deformities in the context of prior IPP implantation surgery. We conducted a retrospective analysis of the Johns Hopkins Hospital institutional database of IPP surgeries from May 2006 to March 2017 consisted of 198 patients who had undergone IPP revisions. All cases involving surgical revisions associated with isolated proximal corporal deformities (proximally from the penoscrotal junction) were identified. Data retrieved included clinical characteristics, operative findings, and surgical outcomes. Main outcome measures are the ability to achieve successful sexual intercourse after IPP revision. The findings of proximal corporal deformities were manifest in fifteen patients who had undergone previous IPP surgery. Deformities consisted of corporal dilatation (15 patients) and corporal rupture (8 patients). Associated prosthesis defects were mechanical failure ( 2 patients), aneurysmal dilatation (5 patients), and device breakage (4 patients). Median IPP treatment duration was 14 years. Mean number of prior IPP surgeries were 4.2 times. All patients underwent reduction corporoplasty with IPP device replacements consisting of controlled expansion devices. Mean age at revision was 59.7 years. All patients reported successful sexual intercourse and satisfaction within 6 months' follow-up after surgical revision. Proximal corporal deformities arising after IPP surgery are associated with dysfunctional device operation and as well as device defects. Proper recognition of this problem allows the opportunity for surgical correction with a definitive reduction corporoplasty. Failure to recognize these abnormalities timely may predispose to unsuccessful revisions and complicate future definitive surgical repair.
\end{abstract}

\section{Introduction}

Penile prosthesis surgery is the standard surgical modality conventionally used for patients who have failed conservative therapy for erectile dysfunction (ED) [1]. The number of patients receiving penile prosthesis devices has steadily increased over the past decade [2]. Common causes of failure after inflatable penile prosthesis (IPP)

Arthur L. Burnett

aburnet1@jhmi.edu

1 Urology Department, College of Medicine, Taibah University, Madinah, Saudi Arabia

2 The James Buchanan Brady Urological Institute and Department of Urology, The Johns Hopkins University School of Medicine, Baltimore, MD, USA implantation necessitating revision include infection, device erosion, and pump migration and, less often, malfunction of the device [3]. Because of improvements in synthetic properties of modern prosthetic devices, more than half of IPP devices are functional beyond 15 years after implantation [4]. The majority of patients requiring surgical revisions beyond ten years present with non-infectious complications, such as mechanical failure or other device functionality problems that result in patient dissatisfaction $[5,6]$.

Reports have described the surgical management and outcomes of latent corporal abnormalities such as extrusion, crossover, and curvatures that may result in compromised device function $[5,7,8]$. These corporal deformities are commonly distal penile defects in nature that are usually clinically evident. We surmised that insidious deformities of the corporal body arising proximally following penile prosthesis surgery also yield suboptimal outcomes. Our 
literature review revealed no previous report describing the management and outcomes of such deformities. In this study, we retrospectively present the natural history and corrective management of failed IPP devices associated solely with proximal corporal deformities.

\section{Materials and methods}

\section{Participants and data collection}

After obtaining institutional review board approval, a retrospective database of all patients who underwent penile prosthesis surgeries at a single institution, the Johns Hopkins Hospital, between May 2006 (when electronic medical recording was commenced) and March 2017 was accrued. Electronic medical records of 733 consecutively eligible patients were reviewed, from which patients who had corporoplasty associated with isolated proximal corporal deformities were identified.

Study eligibility criteria included: (1) corporoplasties at the time of penile prosthesis revision (2) isolated proximal corporal deformities, proximally from the penoscrotal junction. All patients who had undergone corporoplasties for Peyronie's disease, aphallia, corporal fibrosis, corporal erosion, intraoperative corporal perforation, and penetrating traumatic penile injuries were excluded from this study.

\section{Perioperative characteristics and main outcome measures}

Patient demographics and pertinent disease characteristics, including current age, age at first IPP surgery, ethnicity, Charleston comorbidity index and body mass index, prosthesis surgical indications based on clinical evaluation and penile Duplex ultrasound flow study, duration of treatment with IPP devices, and the number of previous IPP revisions were recorded [9]. Perioperative findings including type, location, and extent of proximal corporal deformity, presenting IPP device status, maximal stretched corporal length (MSCL; measured total corporal length during surgery utilizing Dilamezinsert with maximal stretch), and the interval change of MSCL measurements between initial and final IPP surgeries. Primary surgical outcome was assessed as the ability to achieve sexual intercourse without the need for further revision surgery after reduction corporoplasty.

\section{Procedure}

Reduction corporoplasty was done at the time of IPP device removal and replacement in all patients via penoscrotal approach by the same surgeon (ALB), as described previously (Fig. 1) [10]. Proximal neurovascular bundle

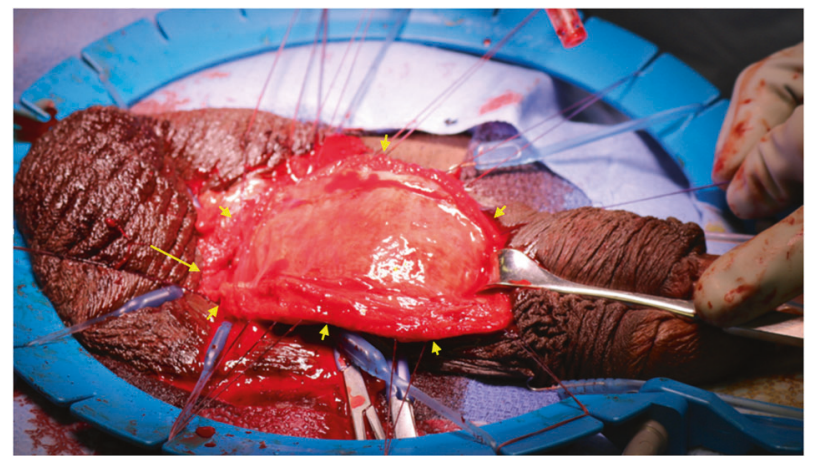

Fig. 1 Photograph of left proximal corporal dilatation after complete dissection. A penoscrotal approach is used. Short arrows indicate the edge of tunica albuginea and long arrow shows the proximal end of the corporal dilatation

preservation was performed with reconstruction of the tunica albuginea whenever necessary. All removed devices were evaluated on a back table to determine associated IPP abnormalities. Prior to device replacement, the Mulcahy salvage protocol was performed [11]. Replaced prosthetic devices were controlled expansion IPP devices (American Medical System [AMS] 700 Controlled Expansion [CX], Minnetonka, MN, USA). IPP devices were activated 6 weeks after procedures.

\section{Statistical analysis}

Descriptive statistics were used to report patient demographics and perioperative characteristics. Mean \pm standard deviation and median (IQR; Interquartile range) were used to describe continuous variables. Frequency and percentage were used to describe categorical variables. Paired student's $t$-test was used for evaluating continuous variables. All statistical analyses were executed using STATA (StataCorp LP, College Station, TX). $P$-values of $<0.05$ were considered statistically significant.

\section{Results}

Among 733 patients who underwent penile prosthesis surgery, a total of 198 (27\%) underwent surgical revisions. Corporal reconstructions were done in $51(25.8 \%)$ patients requiring surgical revisions. Among corporal reconstructions, $15(7.1 \%)$ patients were recognized as having isolated proximal corporal deformities and/or dilatation. The flowchart in Fig. 2 illustrates selection methods among patients. We recognized seven patients with proximal corporal deformities preoperatively; five patients were identified based on clinical presentation (Wobbly Penis) [8], and two patients were known to have structural abnormalities associated with multiple previous corporal reconstructions 
Fig. 2 Flow charts shows patient numbers and selection methods

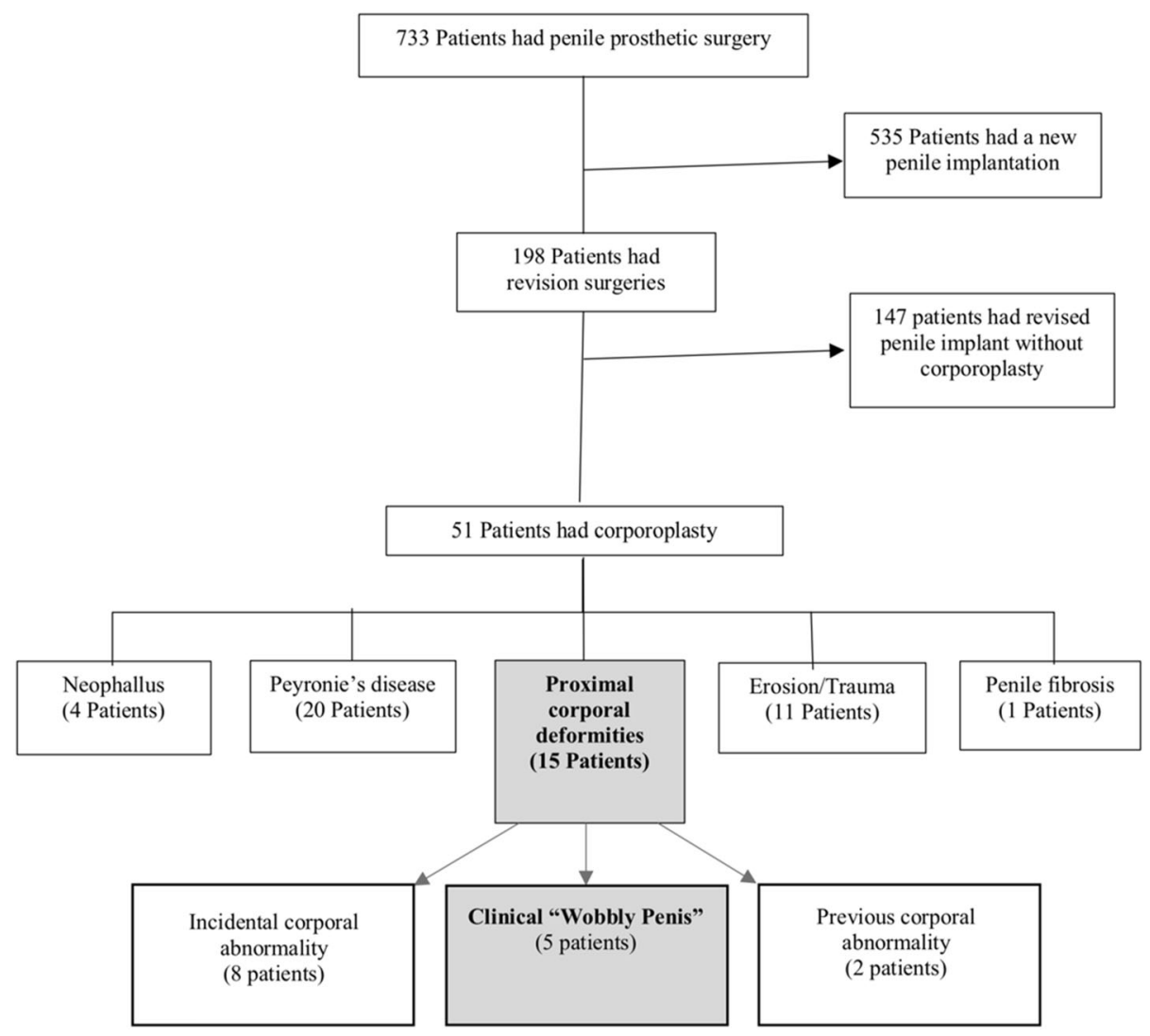

utilizing vascular grafting. We identified eight additional patients with proximal corporal dilatations found incidentally during IPP surgical revisions.

The mean age at the time of presentation was $59.7 \pm 12.1$ years (IQR 35-67). Other baseline clinical characteristics are shown in Table 1. Preoperatively, patients sought medical advice with assorted complaints. Complaints uniformly consisted of instability and mobility of the IPP device within the corporal space and inability to achieve sexual intercourse even for intact and cycling devices. Patients with corporal ruptures described acute penile and deep pelvic pain and were confirmed intraoperatively to have disrupted tunica albuginea. Eight patients had initial IPP placements due to organic ED after failure of conservative therapy with intact anatomical penile integrity. Veno-occlusive dysfunction was assessed in seven patients, among which two patients had undergone previous venous ligation procedures. Other surgical indications are summarized in Table 1 . Ten patients $(66.7 \%)$ had previous IPP revisions with a median of 3 revisions, including a maximal 14 revisions in a patient who underwent his first penile implant surgery at age of 21 years secondary to pelvic trauma.

Auxiliary clinical workup was done in one patient who had delayed intervention whereby his condition worsened with device inoperability. His pelvic magnetic resonance imaging (MRI) study revealed proximal corporal dilatation and prosthetic cylinder aneurysmal dilatation, which was confirmed intraoperatively (Fig. 3).

At the time of reduction corporoplasty, patients underwent IPP replacement with controlled expansion devices. IPP device status at surgery is presented in Table 2. In eight patients, surgical reconstruction for corporal rupture was required. One patient presenting with urethral irritation had an extensive corporal rupture that extended into the urethra; suprapubic bladder drainage and urethroplasty were done prior to corporal reduction and IPP replacement at the same operative setting. IPP expander effects were determined intraoperatively based on corporal length measurements. MSCL data at the time of the first implantation were available in eight patients whose mean initial MSCL was $21.8 \pm 3.3 \mathrm{~cm}$ that increased by a mean $3.4 \pm 2.5 \mathrm{~cm}(p<$ $0.001)$ at reduction corporoplasty. The mean final MSCL for all patients was $25.2 \pm 3.1 \mathrm{~cm}$.

The median follow-up time after reduction corporoplasty was 6 months (IQR 4-24 months). All patients reported successful sexual intercourse after surgical correction. No further revisions were reported. One patient reported decreased glanular sensation secondary to neurovascular mobilization. 
Table 1 Baseline demographic and clinical characteristics

\begin{tabular}{ll}
\hline Patients characteristics & $\begin{array}{l}\text { Mean } \pm \text { SD/Median (IQR) or } \\
\text { number }(\%)\end{array}$ \\
\hline Age at first IPP (years) & $46.9 \pm 16.0$ \\
& $47(37-61)$ \\
Current age (years) & $59.7 \pm 12.1$ \\
& $63(53-67)$ \\
Ethnicity & \\
African American & $9(60 \%)$ \\
Caucasian & $4(26.7 \%)$ \\
Others & $2(13.3 \%)$ \\
Charleston comorbidities index & $3.3 \pm 3.0$ \\
& $2(1-5)$ \\
BMI (kg/m $\left.{ }^{2}\right)$ & $29.2 \pm 5.2$ \\
Surgical indications (initial) & $31.1(26.1-33.6)$ \\
Veno-occlusive ED & $7(46.7 \%)$ \\
Arterial ED & $1(6.7 \%)$ \\
Trauma & $1(6.7 \%)$ \\
Pelvic surgery & $3(20 \%)$ \\
Pelvic radiation & $1(6.7 \%)$ \\
Other & $2(13.3 \%)$ \\
Prior IPP surgical procedures & $4.2 \pm 3.3$ \\
Treatment duration with IPP & $3(2-5)$ \\
device (years) & $13.8 \pm 9.9$ \\
\hline & $14(6-18)$ \\
\hline Br body mass ind & \\
&
\end{tabular}

$B M I$ body mass index, $E D$ erectile dysfunction, $I P P$ inflatable penile prosthesis, $S D$ standard deviation, $I Q R$ interquartile range

\section{Discussion}

In this study, we reported the clinical findings and outcomes after surgical intervention for isolated proximal corporal deformities in fifteen patients. They consisted of proximal dilatation in all patients, as defined by the dimensional difference between the corpus cavernosal space and the prosthetic cylinder circumference, as well as associated conditions such as tunical rupture and IPP device inoperability. We described the natural history of these deformities that included multiple failed IPP device replacements, corporal dilatations and ruptures, and device breakages. Definitive surgical management consisted of reduction corporoplasty and device replacement with controlled expansion IPP devices whereby intact and functional devices were achieved over the follow-up period.

Proximal corporal deformity is a unique IPP surgical complication. It may be challenging to recognize this complication, particularly in patients who present with IPP devices that seem operable. Vigilant clinical assessments followed by axial pelvic body imaging, such as MRI, may facilitate the detection of these penile soft tissues deformities and associated IPP defects in highly suspicious circumstances. We utilized MRI as an adjunctive diagnostic tool in one patient that also aided in surgical planning. Thiel et al. evaluated the use of T2-weighted MRI for different anatomical penile and IPP defects [12]. In this case series, they reported the use of MRI to detect a patient with aneurysmal dilatation and buckling of a prosthesis cylinder due to a preexisting corporal deformity. Preoperative familiarity with the penile wobble effect phenomenon may also assist in recognizing these deformities readily.

Our series revealed several characteristic clinical associations. Patient presentations commonly consisted of gradual loss of device rigidity and penile stability, longstanding IPP duration, and multiple failed IPP revisions. Such presentations exemplify the insidious natural history of this complication that possibly evades prompt recognition and corrective management. Mirheydar and associates performed a retrospective longitudinal analysis of penile prosthetic devices over the long term utilizing a populationbased database from 1995 to 2010 [6]. They found that 14\% of patients were dissatisfied with their device implants even after replacement at long-term follow-up. However, this study was limited by failure to capture detailed descriptions of risk factors in $30 \%$ of patients who sought surgical revision at different centers.

Veno-occlusive ED was prevalent in our cohort and possibly represents a risk marker for corporal structural defects. Chiang and associates compared the tunica albuginea of patients with ED to that of a control group at a microscopic level showing that patients with veno-occlusive ED manifest increased levels of type 3 collagen and noncollagen proteins [13]. Other studies have shown a significant decrease in the number of elastic fibers and structural alterations of the tunica albuginea in patients with $\mathrm{ED}$ associated with impaired veno-occlusive function [14-16]. We acknowledge both known abnormal tunica albuginea structural conditions and proximal dilatation with tunical thinning observed incidentally at the time of IPP revisions in our patient cohort.

During the 1970s, corporal dilatation involving the corporal body in entirety resulted from IPP device overexpansion, representing a common indication for IPP revision surgeries. Furlow and associates reported the occurrence of aneurysmal corporal dilatation in patients who had uncontrolled expansion IPP devices [8]. In their series, the whole corpus cavernosum was dilated but not necessarily at the proximal section as in our cohort. Management of these deformities prior to the introduction of controlled expansion triple-layer cylinders involved wrapping a vascular graft as a reinforcement around prosthetic cylinders at the time of revision. It is noteworthy that two of our patients had been surgically treated with vascular graft 
Fig. 3 MRI appearance of left proximal corporal deformity and cylinder aneurysmal dilatation (AMS 700 LGX). a Sagittal T2 MRI appearance of inflated left IPP cylinder with aneurysmal dilatation. b Intact right cylinder. c Axial T1 inflated images demonstrate proximal corporal wall dilatation
A

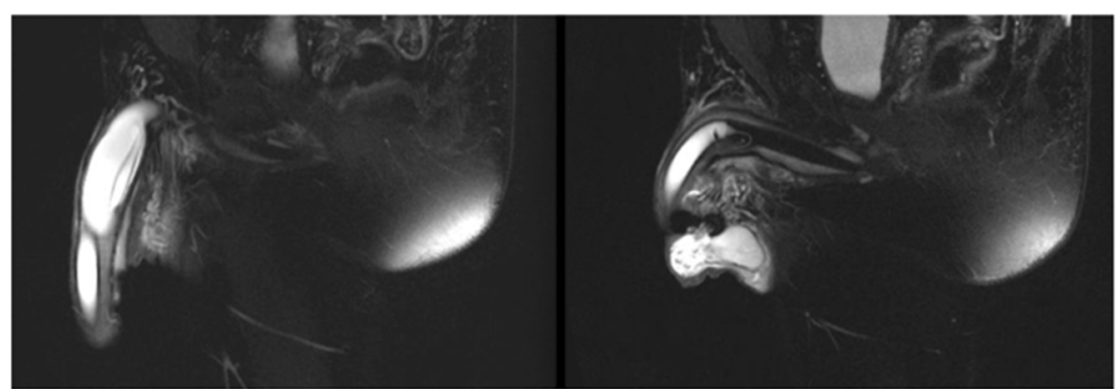

A

B

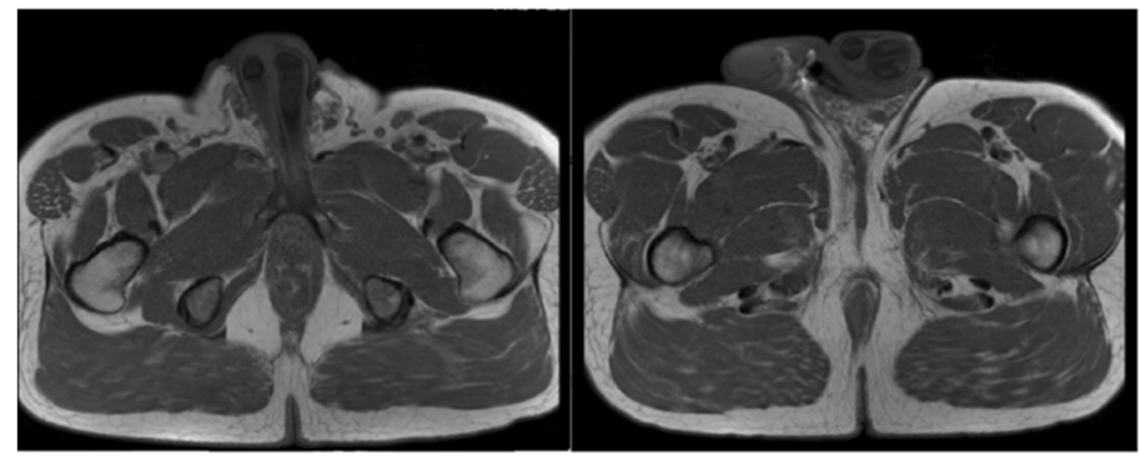

C
Table 2 Operative findings

\begin{tabular}{ll}
\hline Operative characteristics & Mean \pm SD number (\%) \\
\hline Dilatation site & \\
Right & $4(26.7 \%)$ \\
Left & $4(26.7 \%)$ \\
Bilateral & $7(46.7 \%)$ \\
Corporal rupture & \\
Yes & $8(53.3 \%)$ \\
No & $7(46.7 \%)$ \\
IPP status & \\
Intact & $4(26.7 \%)$ \\
Mechanical failure & $2(13.3 \%)$ \\
Aneurysmal dilatation & $5(33.3 \%)$ \\
Device breakage & $4(26.7 \%)$ \\
MSCL & $25.2 \pm 3.1$
\end{tabular}

$I P P$ inflatable penile prosthesis, $M S C L$ maximum stretched corporal length, $S D$ standard deviation, $I Q R$ interquartile range

${ }^{\mathrm{a}}$ Grossly ruptured prosthetic component

wraps yet developed recurrent proximal corporal dilatation at an average of 10 years later.

Mulcahy described the use of controlled expansion devices in 1988 when the triple-layered American Medical System CX device was introduced [17]. The cylinder wall consisted of a middle layer of expandable monofilament polypropylene-like material situated between silicone layers. He addressed the advantage of controlled expansion IPP devices in patients with preexisting thinning of the tunica albuginea to avoid secondary cylinder aneurysmal dilatations. This report supports our finding of five patients who had previously undergone uncontrolled expansion device implantation and subsequently developed cylinder aneurysmal dilatations.

Our study has certain limitations. We acknowledge the relatively small number of patients, which may suggest an unusual occurrence of this problem. The limited sample size hampered further determination and statistical analysis of factors that may have contributed to proximal corporal deformity. Our series is retrospective and comprises patients who had expressed dissatisfaction. It is possible that some degree of corporal dilatation develops for many longstanding IPP devices that is not recognized and goes unaddressed in spite of patient dissatisfaction. Multicenter studies that include evaluation of patient satisfaction may serve to clarify these observations.

\section{Conclusions}

Despite these limitations, this report lends insight into the existence of isolated proximal corporal deformities and associated conditions that prompt awareness and appropriate prompt surgical intervention. Delayed surgical 
intervention and incorrect revision may predispose to device instability, prosthesis cylinder aneurysmal dilatation, prosthesis cylinder breakage, and corporal body/tunical rupture. Proper recognition of this problem before and/or at the time of surgical revision allows the opportunity for surgical correction of this problem with reduction corporoplasty and IPP replacement with a controlled expansion device.

\section{Compliance with ethical standards}

Conflict of interest The authors declare that they have no conflict of interest.

Publisher's note: Springer Nature remains neutral with regard to jurisdictional claims in published maps and institutional affiliations.

\section{References}

1. Hellstrom WJ, Montague DK, Moncada I, et al. Implants, mechanical devices, and vascular surgery for erectile dysfunction. J Sex Med. 2010;7:501-23.

2. http://www.marketresearch.com. Accessed 11 October, 2010.

3. Carson CC, Mulcahy JJ, Govier FE. Efficacy, safety, and patient satisfaction outcome of the AMS $700 \mathrm{CX}$ inflatable penile prosthesis: results of a multicenter study. J Urol. 2000;164:376-80.

4. Wilson SK, Delk JR, Salem EA, Cleves MA. Longterm survival of inflatable penile prostheses: single surgical group experience with 2384 first-time implants spanning two decades. J Sex Med. 2007;4:1074-79.
5. Levine LA, Becher E, Bella A, et al. Penile prosthesis surgery: current recommendation from the International Consultation on Sexual Medicine. J Sex Med. 2016;13:489-518.

6. Mirheydar H, Zhou T, Chang DC, Hsieh TC. Reoperation rates for penile prosthetic surgery. J Sex Med. 2016;13:129-33.

7. Mulcahy JJ. The prevention and management of noninfectious complications of penile implant. Sex Med Rev. 2015;3:203-13.

8. Furlow WL, Motley RC. The inflatable penile prosthesis: clinical experience with a new controlled expansion cylinder. J Urol. 1988;139:945-46.

9. Guzzo TJ, Dluzniewski P, Orosco R, et al. Prediction of mortality after radical prostatectomy by charlson comorbidity index. Urology. 2010;76:553-57.

10. Rajih E, Burnett AL. Penile wobble effect: proximal corporal deformities as a cause of penile prosthesis failure. Sex Med. 2018;6:267-71.

11. Mulcahy JJ. Treatment alternatives for the infected penile implant. Int J Impot Res. 2003;15:147-49.

12. Thiel DD, Broderick GA, Bridges M. Utility of magnetic resonance imaging in evaluating inflatable penile prosthesis malfunction and complaints. Int J Impot Res. 2003;15:155-61.

13. Chiang PH, Chiang CP, Shen MR, et al. Study of the changes in collagen of the tunica albuginea in venogenic impotence and Peyronie's disease. Eur Urol. 1992;21:48-51.

14. Akkus E, Carrier S, Baba K, et al. Structural alterations in the tunica albuginea of the penis: impact of Peyronie's disease, ageing and impotence. Br J Urol. 1997;79:47-53.

15. Sattar AA, Wespes E, Schulman CC. Computerized measurement of penile elastic fibers in potent and impotent men. Eur Urol. $1994 ; 25: 142-4$.

16. Lacono F, Barra S, de Rosa G, Boscaino A, Lotti T. Microstructural disorders of tunica albuginea in patients affected by impotence. Eur Urol. 1994;26:233-9.

17. Mulcahy JJ. Use of CX cylinders in association with 700 inflatable penile prosthesis. J Urol. 1988;140:1420-21. 